\title{
Revisión
}

\section{Traumatismo encefalocraneano moderado: un nuevo enfoque clínico para un término inadecuado}

\author{
Review \\ Moderate Traumatic Brain Injury: A new clinical approach for an \\ inappropriate term
}

\section{Sergio Aguilera Rodríguez', Sebastián Iglesias Vargas²}

${ }^{1}$ Neurocirujano, Servicio Neurocirugía Hospital Clínico Herminda Martin Chillán, Universidad de Valparaíso. Valparaíso, Chile.

${ }^{2}$ Médico Cirujano, Servicio Neurocirugía Hospital Clínico Herminda Martin Chillán, Chile.

\section{Resumen}

El traumatismo encefalocraneano moderado es un grupo de pacientes muy heterogéneo y su mortalidad puede llegar hasta $15 \%$, por lo que el término moderado debería ser reemplazado por traumatismo encefalocraneano potencialmente grave. Su evolución y pronóstico depende de una serie de factores que deben ser evaluados correctamente en la atención hospitalaria inicial del enfermo: edad, puntuación en la Escala de Glasgow, Escala tomográfica de Marshall y tipo de lesión intracraneana. Estos factores se relacionan fuertemente con la evolución del enfermo y las secuelas neurológicas posteriores. Lesiones como la hemorragia subaracnoidea traumática y especialmente la presencia de contusiones cerebrales están asociadas a secuelas neuropsicológicas que pueden ser muy invalidantes para los pacientes. Ahora bien, se deben distinguir al menos dos grupos de pacientes con TEC potencialmente grave: aquellos con Glasgow 11-13 y los pacientes con Glasgow 9-10 puntos. Este último grupo, de alto peligro de mala evolución clínica, si se asocia a una Tomografía Marshall III y IV inicial debieran ser considerados como traumatismo encefalocraneano grave desde la admisión. Un grupo de autores diseñó una categorización "tipo luces del semáforo" utilizando la Escala de Glasgow y la Clasificación de Marshall, lo que facilita reconocer al grupo de pacientes con más riesgo de mala evolución. Dentro del manejo neurointensivo, se debe recalcar la prevención de la hipotensión e hipoxia, la oportuna evacuación de lesiones ocupantes de espacio, el control intensivo de las variables fisiológicas y la neuromonitorización multimodal en el grupo de mayor riesgo. Todas estas medidas lograrán disminuir la mortalidad y mejorar el pronóstico de un grupo de pacientes cuya injuria cerebral primaria no determinó un daño cerebral inicial devastador.

Palabras clave: Clasificación trauma craneoencefálico moderado, contusión cerebral, factores pronósticos, Escala Marshall, neurointensivo.

\section{Abstract}

Moderated TBI (mTBI) is a very heterogeneous group of patients but its mortality is relatively high. For this reason, the term moderated should be replaced by "potencially severe TBI" (psTBI). Outcome depends of prognostic factors that should be correctly considered at the initial hospital evaluation: age, GCS, GCSmotor, Marshall CT classification and type of brain injury as brain contusions or tASH. All these factors are strongly related with in-hospital patient evolution, mortality, outcome and neuropsycological sequels, that are frecuently underrated. In psTBI, we can distinguish 2 groups of patients: those with GCS 11-13 initial, and the GCS $9-10$ patients who are the high risk group for adverse clinical evolution, specially if is associated to Marshall CT category III and IV. This subgroup of patients should be considered as Severe TBI from the

\section{Correspondencia a:}

Sergio Aguilera Rodríguez

aguilera71@gmail.com 
admission. An author's group designed a new categorization using GCS and Marshall Scale creating a traffic light chart that helps to recognize patients with very high risk for neuroworsening. In the neurocritical care, hypotension and hypoxia must be avoided all the time, ocupying mass neurosurgery has to be done early, physiological variables need intensive care and multimodality neuromonitoring in the high risk subgroup should be considered. All these measures will allow improving outcome and decreasing mortality of patients with a not devastating primary brain injury.

Key words: Traumatic brain injury classification, moderated TBI, brain contusion, prognostic factors, Marshall classification, neurocritical care.

\section{Introducción}

Las guías clínicas existentes para el manejo de pacientes con Traumatismo Encefalocraneano (TEC) se han enfocado principalmente en aquellos enfermos con lesiones cerebrales más severas. Sin embargo, existe un grupo de pacientes catalogados como lesión traumática cerebral moderada cuyo resultado funcional e incluso su mortalidad no se condicen con la condición de moderado, resultando con secuelas neurológicas importantes ${ }^{1}$. El objetivo de este artículo es reconocer este subgrupo de pacientes con TEC moderado (TECm) que tienen mayor riesgo de mal resultado final. Identificar qué factores clínicos y radiológicos deben ser considerados en la evaluación del enfermo en la Unidad de Emergencia, la importancia del manejo inicial y neuroquirúrgico, y por qué es fundamental cambiar el paradigma del TEC moderado y comprender que es necesario un nuevo enfrentamiento clínico ante estos casos de "TEC potencialmente grave" (TECpg), en los que tenemos una oportunidad inmejorable de minimizar o prevenir la lesión cerebral secundaria.

Desde mediados de la década de los 70's se ha utilizado la Escala de Coma de Glasgow (GCS, sigla del inglés Glasgow Outcome Score) para clasificar a los pacientes en tres categorías, siendo catalogados como moderados aquellos pacientes que presentan una puntuación entre 9 y 12 puntos ${ }^{2}$. Sin embargo, desde el estudio de $\operatorname{Stein}^{3}$ que demostró $30 \%$ de empeoramiento e incidencia de lesiones intracraneales en pacientes en GCS 13 puntos, es que muchos autores, estudios y guías clínicas han ampliado esta categoría a los casos con GCS entre 9 y 13 puntos. EI TECm y grave según diversas publicaciones fluctúa entre 10 a $15 \%$ del total de pacientes neurotraumáticos y la mortalidad promedio del TECm bordea el $15 \%$ de los casos según diversos autores ${ }^{4}$.

Es fundamental destacar que cerca del $40 \%$ de los pacientes con TEC moderado resultan con discapacidad moderada o severa ${ }^{5,6}$ definida como GOSE $\leq 6$ (sigla del inglés Glasgow Outcome Scale Extended) (Tabla 1).

Muchos de estos pacientes sufren una serie de secuelas neuropsicológicas que pueden resultar muy invalidantes. Todo lo anterior demuestra la importancia de reconsiderar la verdadera gravedad del problema y el desafío que representa este subgrupo de pacientes para todos los equipos de la salud relacionados con la atención de pacientes con lesiones neurotraumáticas. Varios estudios han relacionado las consecuencias neuropsicológicas del traumatismo craneoencefálico con una serie de variables de la fase aguda de la neuroinjuria (Tabla 2).

La recuperación neuropsicológica posterior a un TEC dependerá de la etiología específica de la lesión, su gravedad y localización, así como de la edad del paciente, el nivel intelectual previo a la injuria, e incluso factores genéticos. Pacientes (+) para el alelo 4 de apolipoproteína E tienen mayor riesgo de sufrir enfermedad de Alzheimer relacionado con antecedentes de trauma de cráneo relevante. Entre los trastornos cognitivos más frecuentes y significativos que se pueden encontrar en un paciente que ha sufrido tanto un TEC grave o moderado se pueden describir: Atención y velocidad de procesamiento, Aprendizaje y memoria, Lenguaje y comunicación y Emoción y conducta?.

Estas secuelas que resultan muy invalidantes para el enfermo, generan un costo social y económico significativo, y lo más importante, como veremos más adelante, la magnitud de estas secuelas están en clara relación con la aparición de daño cerebral secundario, que depende del manejo neurocrítico en la etapa aguda de su evolución.

\section{Factores pronósticos}

Una serie de factores están directamente asociados con la evolución y pronóstico funcional final del paciente. Hacer una adecuada interpretación de estos factores pronósticos permitirá tomar decisiones adecuadas, y lo más importante, no subvalorar la gravedad de la lesión neurotraumática, situación que ocurre frecuentemente en los pacientes con

\begin{tabular}{|l|l|}
\hline \begin{tabular}{|l} 
Tabla 1. Escala The Glasgow Outcome Scale Extended \\
Glasgow
\end{tabular} \\
\hline 1 & Outcome Scale Extended (GOSE) \\
\hline 2 & Estado vegetativo \\
\hline 3 & Discapacidad severa inferior \\
\hline 4 & Discapacidad severa superior \\
\hline 5 & Discapacidad moderada inferior \\
6 & Discapacidad moderada superior \\
7 & Recuperación adecuada inferior \\
8 & Recuperación adecuada superior \\
\hline
\end{tabular}


Tabla 2. Variables asociadas a secuelas neuropsicológicas en TECm

Variables asociadas a secuelas neuropsicológicas en TEC moderado

Menor puntuación en la Escala de Glasgow

Duración del compromiso de conciencia

Lapso de amnesia post traumática $>24$ horas

Presencia de contusión cerebral

Carga de hipertensión endocraneana (medido en $\mathrm{mmHg} /$ hora por sobre $20 \mathrm{mmHg}$ )

Insultos sistémicos como hipotensión e hipoxia

TEC malamente catalogados como moderados a su ingreso. No olvidar que la frase "time is brain" también aplica a los pacientes con neurotrauma, y que evitar la lesión cerebral secundaria está directamente relacionado con un manejo neurointensivo oportuno y adecuado.

\section{Edad}

En pacientes adultos con TEC grave y moderado existe una relación lineal entre mayor edad y malos resultados después de los 40 años. Narayan y Heiskanen, encontraron una asociación entre edad mayor de 60 años y casi $80 \%$ mortalidad y mal pronóstico funcional en TEC grave. Estudios focalizados en TEC moderado (Compagnone, 2009) ${ }^{8}$, han demostrado que edad > 60 años sería un factor de riesgo de mal resultado funcional.

\section{Escala de coma de Glasgow}

En TECm, el GCS tiene un espectro muy amplio, desde pacientes algo desorientados y somnolientos $(\mathrm{GCS}=13$ ) hasta pacientes que sólo localizan y balbucean $(\mathrm{GCS}=9)$. En este mismo sentido, se ha demostrado diferencias en la evolución y pronóstico de pacientes con GCS 13 - 11 y aquellos entre $9-10$ puntos $^{8}$. Hasta el $75 \%$ de los fallecidos considerados TECm al ingreso tenían esta puntuación en el GCS ${ }^{4}$. Además, se debe recordar que el apartado motor de la escala de Glasgow (GCSm) tiene casi el mismo valor predictivo que la evaluación completa ${ }^{9,10}$. En este contexto, GCSm $=5$ debe ser considerado un factor de alarma y más aún, considerar GCSm = 4 como un paciente grave. Hacer estas sutiles distinciones ayuda a no subestimar la eventual gravedad de una injuria cerebral traumática desde el ingreso.

\section{Características tomográficas}

La presencia de lesiones focales > $25 \mathrm{ml}$, la compresión o ausencia de cisternas basales y la desviación de la línea media se han asociado a mala evolución en TEC grave y moderado ${ }^{11}$. Ahora bien, en los pacientes con TECm, se debe considerar además la presencia de hemorragia subaracnoidea traumática, las contusiones cerebrales y la presencia de fracturas con hundimiento, dado que todas éstas se asocian a mayor riesgo de mal pronóstico funcional en pacientes que ingresan en GCS $9-13$, especialmente secuelas neuropsicológicas como se describió anteriormente. Cabe mencionar además que las cisternas basales son un factor independiente de mal pronóstico y riesgo de hipertensión endocraneana pero no sólo su ausencia (Marshall III) si no también la compresión de éstas, subdivisión que podemos encontrar en la escala Tomográfica de Rotterdam ${ }^{12}$. Esta es una distinción que se debe reconocer al momento de interpretar la tomografía de un paciente con neurotrauma (Figura 1).

\section{Lesión axonal difusa}

Constituye una lesión provocada por daño axonal parcial o total, secundario o primario en la sustancia blanca. Puede reconocerse como pequeñas lesiones hiperdensas que corresponden a lesiones hemorrágicas, aunque pueden pasar desapercibidas en una tomografía y ser mejor evaluadas con algunas secuencias de Resonancia Magnética. Se puede clasificar en tres grados (Adams, 1989): Grado 1: Lesiones en sustancia blanca de los hemisferios cerebrales. Grado 2: Grado 1 más lesiones en cuerpo calloso (más frecuente en el esplenio, asociado a hemoventrículo variable) y Grado 3: Grado 2 más lesión ubicada en mesencéfalo ${ }^{13}$.

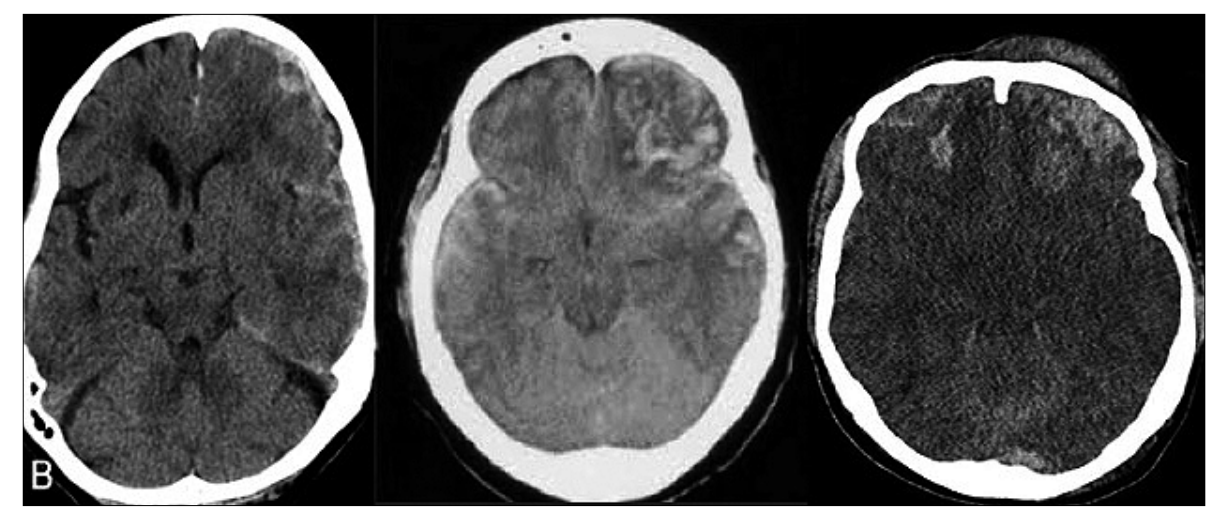

Figura 1. Imágenes de tomografía cerebral, que evidencia de izquierda a derecha cisternas basales normales, comprimidas y ausentes, respectivamente. 


\section{Biomecánica de la lesión}

Se debe valorar la cinemática del trauma y sus efectos sobre el encéfalo. En general, los mecanismos que involucran fenómenos de aceleración y desaceleración provocan injurias cerebrales más significativas. En distintas publicaciones, los pacientes que ingresan como TECm asociados a peor pronóstico sufrieron accidentes de tránsito, atropellos y caídas de altura.

\section{Lesiones asociadas}

La prevalencia de lesiones extracraneanas en pacientes con neurotrauma varía entre $23 \%$ y $41 \%$. Traumas con Injury Score Severity (ISS) $\geq 15$ puntos, se asocian a mal resultado en los pacientes con TECm, así lo demostró Fabbri ${ }^{14}$, determinando que en pacientes con resultados desfavorable, el $73 \%$ presentaba un ISS >15 vs $42 \%$ en aquellos con buen resultado. Es muy probable que la triada politrauma, manejo subóptimo en la hora dorada (especialmente hipotensión arterial e hipoxia) y la aparición de lesiones secundarias se relacionan con la mala evolución de pacientes con TECm.

\section{Contusiones cerebrales}

Las contusiones cerebrales (CC) son lesiones parenquimatosas evolutivas causadas por la energía cinética liberada por el trauma mediadas por varios mecanismos biológicos que han sido objeto de estudio en los últimos años y están asociadas a empeoramiento clínico y a un mal resultado final. Según el trabajo de Kurland ${ }^{15}$, el daño primario provocado por el trauma lleva a la disrupción de neuronas, astrocitos y oligodendrocitos causando necrosis celular. Además, provoca la rotura de vasos sanguíneos y extravasación hemática.

La lesión secundaria, provocada por las alteraciones microvasculares desencadenadas en la lesión primaria lleva a diversas consecuencias: 1 ) isquemia tisular debido a alteración del flujo sanguíneo; 2) edema de la unidad glioneurovascular, y 3) pérdida de integridad y disrupción de otros vasos sanguíneos. Todo esto resultará en la progresión de la lesión hemorrágica, hipertensión endocraneana y mayor isquemia tisular.

Los fenómenos moleculares que determinan la progresión de las CC comienzan con la activación de factores de transcripción mecanosensitivos Sp1 y NF-kB que determinan el aumento de la expresión de subunidades SUR-1 (sulfonilurea tipo 1) que junto a la subunidad TRPM4 forman canales que permiten el ingreso de sodio, calcio y $\mathrm{H}_{2} \mathrm{O}$ a la célula provocando edema intracelular y necrosis en neuronas, glía y células endoteliales, lo que provocará fragmentación capilar, extravasación del contenido hemático y progresión de la lesión hemorrágica hacia las áreas pericontusionales. La sobreexpresión de SUR-1 también se asocia a la presencia de hipoxia-isquemia tisular, por lo que su aparición amplificará el daño tisular cerebral.

Por otro lado, el edema cerebral asociado a las contusiones cerebrales está mediado por la actividad de canales de agua conocidos como Aquaporinas, los que se encuentran ampliamente distribuidos en el cerebro y juegan un rol fundamental en el transporte de agua a través de la membrana celular. Se ha demostrado que 2 horas después de la injuria, existe un aumento en la expresión de Aquaporina 4 a nivel de la Barrera Hemato-Encefálica (BHE) (células endoteliales y astrocitos) generando un aumento de la permeabilidad e incluso la disrupción de la misma.

Finalmente, se deben mencionar las metaloproteasas (MMP), endopeptidasas extracelulares implicadas en la disrupción de la $\mathrm{BHE}$, expansión de contusiones cerebrales y edema cerebral en pacientes con TEC ${ }^{16}$. Se ha demostrado un aumento de niveles de MMP-9 en zonas pericontusionales dentro de las primeras 24 horas de evolución. Las MMPs destruyen las uniones estrechas entre células endoteliales por degradación de proteínas como la claudina y ocludina. La mayor síntesis de estas enzimas se asocia a la activación de la microglia, la presencia de citoquinas (IL-1B) y factores de crecimiento (TGF- $B$ y VEGF), entre otros.

El pronóstico de los pacientes con contusiones cerebrales dependerá de la localización y extensión del daño cerebral irreversible, el que estará relacionado con la magnitud del daño primario y la presencia y severidad del daño secundario. En general, son más frecuentes en el lóbulo frontal y temporal (cerca del $80 \%$ ). En la TC se pueden presentar una lesión hiperdensa, mixta, o con el típico patrón "en sal y pimienta", rodeado por un halo hipodenso que corresponde al área pericontusional. También debe ser tomado en cuenta el concepto de laceración cerebral que en la TC se aprecia como una lesión hemorrágica intraparenquimatosa evacuada hacia el espacio subdural por rotura de la piamadre.

En este tipo de lesiones, el tiempo pasa a ser un factor fundamental, ya que una contusión cerebral es una lesión evolutiva. La cada vez más rápida atención prehospitalaria y el estudio imagenológico precoz (antes de 4 horas) puede subestimar una contusión cerebral inicial. Siempre se debe considerar repetir la tomografía en presencia de lesiones hemorrágicas durante las primeras 6 a 24 horas transcurridas desde el primer examen, incluso en pacientes estables desde el punto neurológico. Según el estudio de Oertel ${ }^{17}$, hasta el $49 \%$ de las lesiones hemorrágicas intraparenquimatosas tienden a aumentar de volumen durante las primeras horas de evolución.

\section{Clasificación tomográfica de Marshall}

La clasificación de Marshall ${ }^{11}$ se diseñó a partir del Traumatic Coma Data Bank (TCDB) y permite una evaluación imagenológica rápida y sistemática del paciente con TEC. Si bien es cierto fue desarrollada en pacientes con TEC grave, esta clasificación es extrapolable a los pacientes con TEC moderado. Junto a la edad y GCS de ingreso, son factores predictivos del pronóstico del paciente. El análisis de la tomografía consta de la evaluación de tres características que se pueden reconocer fácilmente: 1) presencia de lesiones focales hemorrágicas (hiperdensa) mayor de $25 \mathrm{ml}$; 2) presencia vs ausencia de las cisternas perimesencefálicas y 3) desviación de la línea media mayor a $5 \mathrm{~mm}$. Dentro de las falencias de la Escala de Marshall se puede mencionar que no considera el carácter evolutivo de las lesiones, el hecho de tener categorías muy amplias, como la lesión difusa tipo II y la lesión focal evacuada, que puede incluir lesiones con un pronóstico y manejo muy diferentes. Sin embargo, la importancia de esta clasificación, es que permite identificar a aquellos pacientes que tienen mayor riesgo de desarrollar hipertensión endocraneana durante su evolución, como lo demuestra el estudio de M. Poca y cols ${ }^{18}$ 
sobre 94 pacientes (Tabla 3).

Por lo tanto, un paciente con una lesión difusa tipo III o IV deben considerarse un paciente de alto riesgo de mala evolución neurológica a pesar de ser catalogado como TEC moderado según la escala de Glasgow, más aún en pacientes con GCS 9 o 10, GCSm $\leq 5$ y/o si tiene más de 60 años.

\section{Manejo inicial del paciente con TEC potencialmente grave (TECpg)}

Bajo la consigna "tiempo es cerebro", en el TECpg el neurorescate debe comenzar en el lugar del trauma. Durante el período prehospitalario la hipotensión e hipoxia son los dos factores sistémicos de injuria secundaria más importantes. En Chile, las guías clínicas del MINSAL $^{19}$ recomiendan el manejo según el algoritmo de la ATLS a todos los pacientes evaluados en GCS 12 o menos con el objetivo de evitar la lesión cerebral secundaria.

Ahora bien, la hipotensión durante el prehospitalario definido como presión arterial sistólica (PAS) $<90 \mathrm{mmHg}$ es un concepto que debe ser reconsiderado. Hay publicaciones que demuestran que existe una relación continua entre mayor riesgo de mortalidad y mala evolución progresiva con valores de $\mathrm{PS}<120 \mathrm{mmHg}^{20}$, por lo mientras sea posible, evitar valores de presión sistólica bajo $120 \mathrm{mmHg}$ parece una recomendación razonable. En los pacientes con neuroinjuria severa, muchas veces la autorregulación se altera por lo que el flujo sanguíneo cerebral (FSC) es dependiente de la presión arterial media (PAM), y por otro lado, trabajos clásicos de Bouma y Marion demostraron un estado de bajo FSC en las primeras horas post trauma. Recordar por último, que la reposición de fluidos debe ser realizada con solución fisiológica, y no se debe usar soluciones glucosadas en los pacientes con lesión cerebral.

Por otro lado, dentro de la tríada básica a considerar en el manejo inicial, no se puede dejar de mencionar la importancia de la normoventilación durante las primeras horas. Mantener una $\mathrm{pCO}_{2}$ entre 35 - $40 \mathrm{mmHg}$ es fundamental para evitar la lesión secundaria cerebral. Conocidos son los efectos deletéreos de la hipocapnia, la que provoca vasoconstricción arteriolar (hipoxia tisular isquémica), desviación de la curva de disociación de la hemoglobina $(\mathrm{Hb})$ hacía a la izquierda (hipoxia por baja extractabilidad) y aumento de la entrada de calcio a la célula (favoreciendo el daño celular). Se ha demostrado la clara asociación entre valores $\mathrm{pCO}_{2}$ fuera de rango durante el prehospitalario y su efecto sobre la sobrevida en pacientes con TEC 21 .

Por lo anterior, las bases fisiopatológicas en las que se sustenta el manejo inicial de los pacientes con neuroinjuria deben ser consideradas para tomar las medidas que aseguran la prevención del daño cerebral secundario; mucho se ha escrito y dicho sobre la "Hora Dorada" del paciente traumático, sin embargo, seguimos viendo frecuentemente manejos subóptimos que afectan el resultado funcional del enfermo. El control periódico y estricto de los parámetros fisiológicos más básicos son las medidas más eficientes para evitar lesiones secundarias durante el prehospitalario.

Paralelo a la reanimación inicial, la evolución neurológica del paciente es primordial, y en este punto, uno de los problemas más frecuentes es la falta de registro de la evolución de la puntuación de Glasgow. Para neurocirujanos e intensivistas es fundamental conocer su evolución en el tiempo, así como el adecuado registro de las drogas utilizadas en caso de intubación o sedación. A menudo, se toman malas decisiones por la falta de este tipo de información. Por otro lado, se debe actuar rápidamente frente a pacientes que cursen con neurodeterioro, es decir, caída de 2 o más puntos en el GCS, 1 punto GCSm, o la aparición de anisocoria y respuesta motora anormal ${ }^{22}$. Siempre recordar que la primera medida es reevaluar rápidamente el $\mathrm{ABC}$, asegurar la vía aérea si procede, corregir episodios de hipotensión o hipoxia si es necesario, y posteriormente, manejar de forma empírica la hipertensión endocraneana. Para ello, el uso de soluciones salinas hipertónicas (independientes de la concentración usadas en cada centro) son la primera indicación por sobre el Manitol, dado que las soluciones salinas mejoran la PAM y disminuyen la presión intracraneal (PIC). En una emergencia neurológica como ésta, el último recurso es el uso de hiperventilación ( $\mathrm{pCO}_{2}$ nunca menor a $25 \mathrm{mmHg}$ ) por períodos breves de tiempo en espera de la tomografía cerebral.

\section{Manejo neuroquirúrgico}

El manejo neuroquirúrgico es uno de los pilares fundamentales en evitar un peor resultado en pacientes con TECpg de alto riesgo de mala evolución clínica. Según diversos estudios, la incidencia de lesiones intracraneanas varía entre $30 \%-60 \%{ }^{23}$, incidencia que aumenta en pacientes de mayor edad llegando incluso al $80 \%$ de los pacientes mayores de 65

Tabla 3. Tipo de lesión según Clasificación de Marshall, incidencia de HTEC y malos resultados

\begin{tabular}{|lcc|}
\hline \multicolumn{2}{|c|}{ Tabla 3. Tipo de lesión según Clasificación de Marshall, incidencia de HTEC y malos resultados } \\
\hline & Incidencia HTEC (\%) & Malos resultados (\%) \\
\hline Lesión difusa tipo I & - & 0 \\
\hline Lesión difusa tipo II & $28 \%$ & $33 \%$ \\
Lesión difusa tipo III & $63 \%$ & $55 \%$ \\
\hline Lesión difusa tipo IV & $100 \%$ & $100 \%$ \\
Lesión focal evacuada (V) & $65 \%$ & $52 \%$ \\
\hline Lesión focal no evacuada VI & $84 \%$ & $77 \%$ \\
\hline Tomado de M.A. Poca. & & \\
\hline
\end{tabular}




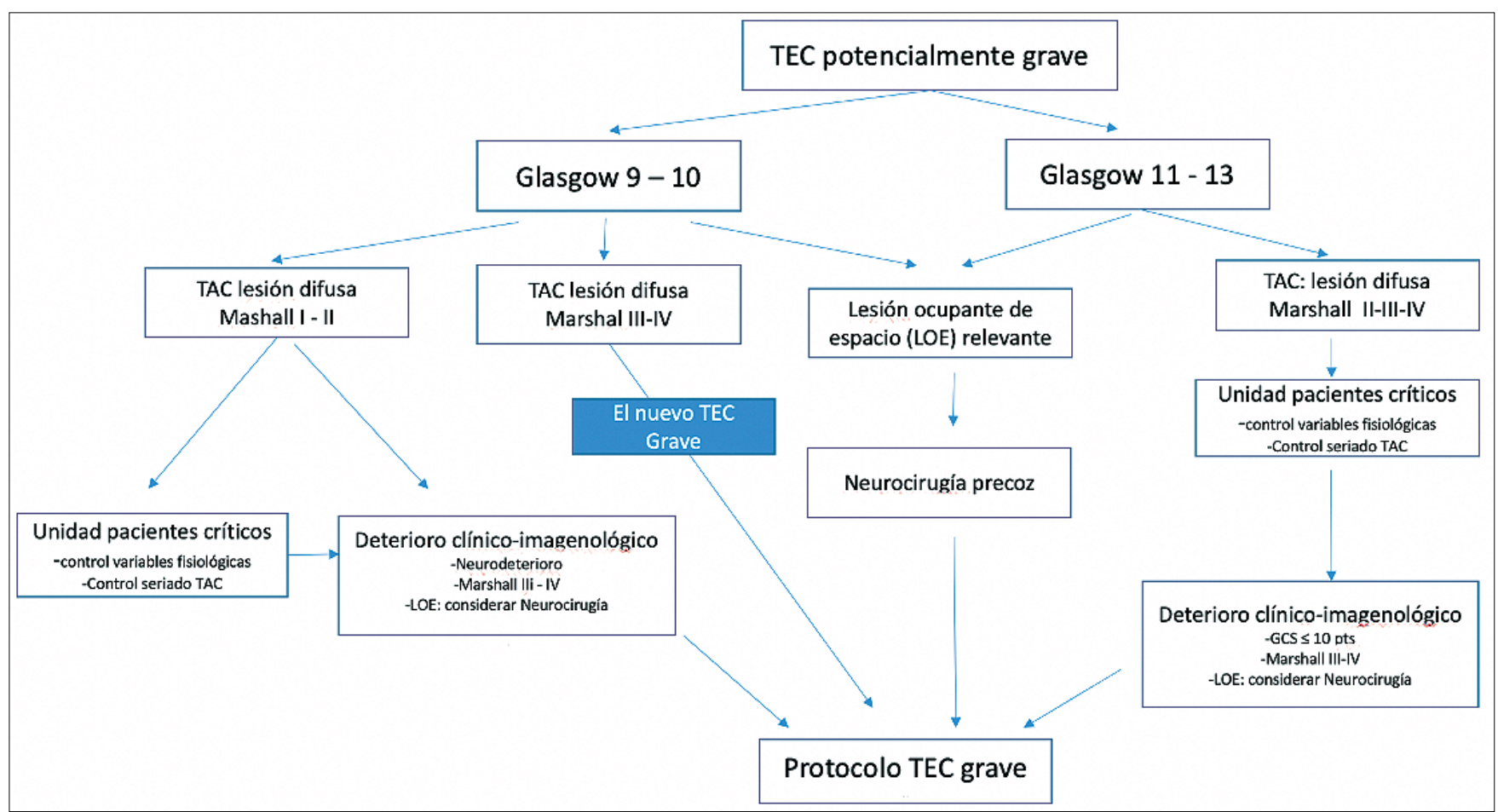

Figura 3. Propuesta de algoritmo de actuación frente a pacientes con TECpg una vez estabilizado en la sala de emergencia. Nótese que los pacientes en GSC 9-10, con TC Marshall III y IV desde un comienzo deberían ser tratados como un TEC grave.

años. En el estudio de Fabbri' ${ }^{14}$, la intervención neuroquirúrgica en los casos necesarios, resultó ser un factor asociado a buen pronóstico $(\mathrm{OR}=0,03,95 \% \mathrm{Cl} 0,01-0,15 ; \mathrm{p}=0,001)$, por lo que, en el manejo inicial del enfermo, una vez hecha la evaluación clínica y tomográfica, se debe considerar la evacuación precoz de lesiones ocupantes de espacio, situación que ocurre en aproximadamente $9-22 \%$ de los casos. Hematomas extradurales $>30 \mathrm{ml}$ volumen o $10 \mathrm{~mm}$ de espesor y subdurales de más de $10 \mathrm{~mm}$ espesor y/o desviación línea media > $5 \mathrm{~mm}$, son indicaciones quirúrgicas ampliamente aceptadas y forman parte de las recomendaciones de las guías clínicas en nuestro país.

En relación a las contusiones cerebrales, la literatura es escasa y no concluyente, sin embargo, existen algunas recomendaciones disponibles en la literatura. El objetivo de la cirugía será prevenir el daño cerebral secundario, dado el efecto neurotóxico e inflamatorio de los productos hemáticos extravasados ${ }^{17}$, y por otra parte, se debe considerar que el core de la contusión es tejido no viable, por lo que, su resección no aumentará la pérdida de tejido cerebral. Las guías quirúrgicas en TEC (Bullok, 2006), recomienda la evacuación quirúrgica de lesiones intraparenquimatosas mayores de 20 cc en pacientes con GCS 6 - 8 puntos y signos de hipertensión endocraneana en la tomografía, y de toda lesión mayor de 50 cc de volumen ${ }^{24}$.

En el trabajo de Mendelow (STITCH, 2015) se analizaron 171 pacientes (más de $50 \%$ en pacientes con TECm) y se estudió el efecto de la cirugía precoz de contusiones cerebrales en el outcome ${ }^{25}$. El $63 \%$ de los pacientes con tratamiento quirúrgico resultaron con buen pronóstico, frente a $53 \%$ de aquellos con tratamiento médico (OR: 0,$65 ; p=$
0,171). Si bien la diferencia en el outcome no alcanzó significancia estadística, la mortalidad entre ambos grupos sí fue significativa ( $15 \%$ vs $33 \%$; $p=0,007)$. Los pacientes con GCS 9 - 12 fueron los que presentaron mejor respuesta a la cirugía precoz. La decisión quirúrgica tardía en pacientes con manejo conservador inicial fue $36 \%$ de los enfermos, un tercio de ellos dentro de las primeras $12 \mathrm{~h}$ post randomización. Las causas del cruce fue el deterioro neurológico (caída del Glasgow, alteración pupilar), progresión del tamaño de la lesión, signos tomográficos (desviación de la línea media), y/o aumento de la PIC. A pesar de la decisión quirúrgica, los pacientes que se deterioraron y fueron a una cirugía tardía tuvieron peor resultado final (GOSE $1-2-3=65 \%$ vs 33\%). Por lo tanto, la decisión y el tiempo de la cirugía en lesiones ocupantes de espacio en pacientes con TECpg tiene un impacto significativo en la evolución posterior del paciente y su resultado funcional final.

\section{Manejo neurocrítico}

El manejo médico de los pacientes con TECm no dispone de guías clínicas específicas y la razón de aquello es la escasa bibliografía y que la evolución de este grupo de pacientes incluye un amplio abanico de posibilidades. Sin embargo, un objetivo común es evitar la lesión cerebral secundaria, y para ello, es fundamental mantener la homeostasis cerebral. Por lo tanto, aunque parezca obvio, las medidas más básicas si consideramos el Neurointensivo serán las más importantes en la etapa aguda. Normovolemia a expensas de solución salina fisiológica y mantener una $\mathrm{SatO}_{2} \geq 95 \%$. Los pacientes con lesión cerebral traumática tienen aumento de las demandas metabólicas por lo que, la alimentación debe ser 
iniciada tan rápido como sea posible y el aporte nutricional completo debe alcanzarse entre el $5^{\text {to }}$ y $7^{\text {mo }}$ día de evolución (BTF guidelines, 2016). El adecuado tratamiento del dolor juega un rol importante en pacientes con lesión cerebral traumática dado que esto genera una respuesta simpática, aumento de la PAM, puede provocar agitación psicomotora y consecuentemente un aumento de la PIC. En estos pacientes es preferible la administración de analgesia en forma continua, evitando los fármacos que puedan alterar la evaluación neurológica. Por otra parte, la agitación en un paciente durante las primeras horas debe más bien ser interpretado como un signo de alarma y antes de iniciar sedación se debe descartar la aparición o evolución de lesiones intracraneales con una tomografía de control. El manejo estricto de la glicemia debe ir orientada a mantener niveles de glicemia $<200$ $\mathrm{mgs} / \mathrm{dl}$. Se ha demostrado que esquemas más agresivos de control glicémico se asocian a mayor incidencia de valores de glucosa cerebral bajos y episodios de crisis metabólicas cerebrales $^{26}$ detectadas con microdiálisis cerebral. En todo paciente con TEC grave y moderado, el correcto manejo del sodio es fundamental. Se deben evitar niveles de sodio bajo $135 \mathrm{mEq} / \mathrm{L}$ y por el contrario, tolerar hipernatremias leves. La temperatura cerebral en condiciones normales supera en promedio $1^{\circ} \mathrm{C}$ a la temperatura central, sin embargo, en un cerebro lesionado esta diferencia puede superar los $2^{\circ}$ $\mathrm{C}$, por lo que el control térmico en los pacientes neurotraumáticos es relevante. La fiebre es común en pacientes con lesión cerebral traumática y se asocia a mayor estadía en unidad cuidados intensivos (UCI) y a peor pronóstico (Jiang, 2002). Se ha demostrado que la hipertermia puede generar lesión axonal secundaria. Se debe considerar la prevención y el manejo precoz de la fiebre en todo paciente que curse con una lesión cerebral. El antipirético de elección no está definido por lo que se debe usar el fármaco disponible en cada unidad para evitar temperatura $>37,5^{\circ} \mathrm{C}$. No existe una recomendación formal en relación al beneficio de iniciar terapia anticonvulsiva en pacientes con TECm. Recordar que en TEC grave, las guías BTF sólo recomiendan su indicación por una semana, y que su uso no previene la aparición de epilepsia tardía (Carney, 2016). Por otro lado, está demostrado que el uso de fenitoína (el fármaco más disponible y usado) retrasa los progresos en la neurorehabilitación ${ }^{27,28}$ y que los efectos neuroprotectores del levetiracetam no están demostrados cabalmente.

Ahora bien, ¿Afectan las alteraciones de la homeostasis fisiológica el resultado del paciente? La respuesta a esta pregunta queda respondida en un interesante trabajo de Lund que muestra la evolución de variables fisiológicas, su relación con la estadía en $\mathrm{UCl}$ y el resultado final en pacientes precisamente con $\mathrm{TECm}^{29}$. Se estudiaron 119 pacientes, 84\% de ellos ingresó a UCI desde el primer día, y 51\% tuvo una estadía $\geq 3$ días. Aquellos pacientes con $>3$ días en $\mathrm{UCI}$ presentaron menor puntuación GCS (13 vs 12), más lesiones extracraneanas, más episodios de hipotensión, hipoxia, hipertermia, anemia e hiperglicemia. De los pacientes que estuvieron más días en UCI evolucionaron con discapacidad $49 \%$ (definido como GOSE $\leq 6$ ), frente a $26 \%$ de los que estuvieron $0-2$ días ( $p=0,017$ ). Por lo tanto, en pacientes con TECm, el ingreso a una unidad de cuidados intensivos, la monitorización de las variables fisiológicas y el tratamiento intensivo de las variaciones no sólo asegura disminuir la aparición de lesión cerebral secundaria sino que también disminuiría el riesgo de mala evolución clínica final.

\section{Neuromonitorización avanzada}

Respecto a la neuromonitorización en TECm, no existen recomendaciones. Esto dependerá de la evolución clínica y radiológica del paciente en las primeras 48 horas. En relación a la PIC, existen una serie de publicaciones respecto a su medición en pacientes con TECm (Stein, Andriessen, Fearnside). Lo interesante de estos estudios resumidos en la Tabla 4, es que en promedio se decidió la monitorización en alrededor de $10 \%$ de los casos, y la PIC se encontraba elevada en cerca del $50 \%$ de los pacientes ${ }^{30,31}$.

Respecto a la monitorización de la oxigenación cerebral a través de la presión tisular de oxígeno $\left(\mathrm{ptiO}_{2}\right)$, tampoco existe evidencia relevante. Sin embargo, cabe mencionar el recientemente publicado estudio BOOST-II6 que comparó el manejo de pacientes sólo con monitorización de la PIC vs PIC más $\mathrm{ptiO}_{2}$. Este estudio incluyó pacientes que presentaron deterioro neurológico dentro de las primeras 48 horas. Lo interesante es que este trabajo concluyó que el tratamiento de pacientes según los valores de $\mathrm{PIC}+\mathrm{ptiO}_{2}$ disminuyó la incidencia de hipoxia tisular cerebral y mostró una tendencia hacia una menor mortalidad y más favorable resultados que aquellos pacientes en lo que sólo se trató la PIC. Sin embargo, aún se debe realizar el estudio fase III para evaluar el real impacto en el pronóstico de la monitorización con PIC $+\mathrm{ptiO}_{2}$.

\section{Direcciones futuras}

Como se ha mencionado, la fisiopatología del neurotrauma es complejo. Por esta razón, es difícil desarrollar modelos para determinar la evolución y el pronóstico de los pacientes desde una etapa temprana. Los biomarcadores son moléculas que pueden reflejar procesos fisiológicos y patológicos. Biomarcadores específicos de lesión cerebral aguda pueden ayudar a categorizar el riesgo de progresión y evolución del enfermo y también predecir el pronóstico a largo plazo, por lo que los niveles en sangre de éstos aparecen como una útil herramienta diagnóstica y de neuromonitoreo ${ }^{32}$. Varios de estos son marcadores específicos de origen neuronal, glial y axonal reflejando los distintos mecanismos de neuroinjuria. El nivel inicial y el perfil temporal de un panel de biomarcadores probablemente dependerá de la severidad del daño cerebral. S100-beta, NSE, GFAP, UCH-L1 y NfL son los biomarcadores más estudiados hasta este momento ${ }^{33,34,35}$. En los próximos años, un panel de biomarcadores será muy útil junto a la Escala de Glasgow, la clasificación tomográfica y otros factores pronósticos en reconocer y subdividir a los pacientes en la etapa inicial de un TEC potencialmente grave para determinar aquellos que tengan más riesgo de deterioro clínico y peor resultado funcional (Tabla 5).

\section{Enfrentamiento clínico de un término inadecuado}

¿Son todos los TEC moderados iguales?, el término 
moderado es inexacto y vago ${ }^{36}$. Como se mencionó anteriormente, esta categoría incluye un espectro muy amplio. Desde el punto de vista clínico, se pueden reconocer al menos 2 grupos de pacientes, aquellos enfermos que tienen un GSC inicial entre 11 y 13 puntos y los que presentan una puntuación entre 9 y 10 puntos. Este último grupo, son pacientes que evolucionan con mayor deterioro clínico, son víctimas de traumas de mayor energía y su desenlace está asociado a peor outcome final como lo describió Compagnone y otros autores.

Por esta razón, se diseñó una matriz visual "tipo luces de semáforo", combinando Escala de Glasgow y la clasificación tomográfica de Marshall ya descrita; esto resulta en un matriz de fácil interpretación que permite poner énfasis en aquellos pacientes con mayor riesgo de mala evolución clínica $^{37}$. Pacientes en categoría Amarillo, de alto riesgo de deterioro, deben ser monitorizados de forma clínica y radiológica seriada estricta, idealmente en una unidad de pacientes críticos. Por otro lado, aquellos en categoría Roja son pacientes de inminente peligro de neurodeterioro que requieren cuidados intensivos y neuromonitorización según las herramientas (invasivas o no invasivas) disponibles en cada centro. Se debería considerar esta categorización una vez estabilizado el paciente, y la decisión debe ser tomada en conjunto entre Emergenciólogos, Neurocirujanos e Intensivistas (Figura 2).

En este mismo sentido, como se plantea en el algoritmo propuesto más abajo para pacientes con TECpg, se podrían tomar en cuenta algunas consideraciones:

- Neurocirugía precoz de lesiones con indicación de resorte quirúrgico.

- $\quad$ En pacientes en GCS 9 - 10 no esperar mayor deterioro clínico es fundamental.

- Considerar la evacuación quirúrgica de lesiones ocupantes de espacio en pacientes con GCS 13 - 11 que presenten deterioro clínico y/o imagenológico.

- En el postoperatorio, incluir a todos los pacientes en protocolo de TEC grave, a excepción de algunos pacientes con hematoma extradural aislado.

- En pacientes con lesiones difusas el control estricto de las variables fisiológicas debe ser intensivo, y el control tomográfico seriado al menos durante las primeras 48 horas.

- Considerar neuromonitorización según los recursos disponibles y cuidados intensivos en aquellos enfermos GCS 11-13 con lesión difusa II, III y IV y aquellos pacientes GCS 9-10 con lesión difusa I y II que presenten deterioro clínico -imagenológico.

- Los pacientes GCS 9 -10 con lesión difusa Marshall III y IV deben ser manejados con protocolo de TEC grave desde el ingreso. Este grupo de pacientes, debería ser incluido en una redefinición del TEC grave.

\section{Conclusiones}

El término TECm es inadecuado dado que representa un grupo de pacientes muy heterogéneo, de evolución incierta, pero que nos ofrece una única oportunidad de evitar secuelas asociadas a lesión cerebral secundaria si se toman las decisiones correctas. Para ello es necesario conocer los factores pronósticos asociados a mala evolución clínica desde el ingreso del paciente. La combinación entre la Escala de Glasgow y la Clasificación Tomográfica de Marshall permite una categorización tipo semáforo, de fácil interpretación y alto impacto visual que permite diferenciar pacientes con distinto riesgo de mala evolución. Aquellos en categoría rojo deberían ser considerados como pacientes con alto peligro de desarrollar neurodeterioro durante su evolución. Pacientes en categoría amarillo y rojo deben ser manejados en unidades de pacientes críticos desde el ingreso hospitalario. Desde el punto de vista neuroquirúrgico, la evacuación de lesiones ocupantes de espacio relevantes debe ser precoz dado que se asocia a mejores resultados, en especial en el grupo pacientes con GCS 9-10. El enfrentamiento clínico más agresivo en este grupo de pacientes con TECpg nos permitirá evitar y/o minimizar la aparición de lesión cerebral secundaria y por ende, disminuir los malos resultados y las secuelas moderadas y graves. Para ello es necesario modificar los conceptos relativos a la gravedad de la lesión traumática usando las mismas herramientas de evaluación que disponemos actualmente. En este contexto, los pacientes con GCS 9-10 y lesión difusa Marshall III y IV deberían ser incluidos en una nueva definición de TEC grave (Figura 3).

\section{Referencias}

1. Andriessen TM et al. Epidemiology, severity classification, and outcome of moderate and severe traumatic brain injury: a prospective multicenter study. J Neurotrauma. 2011;28:2019-31.

2. Teasdale G, Jennett B. Assessment of coma and impaired consciousness. A practical scale. Lancet 1974; 2: 81-4.

3. Stein SC, Ross SE. Moderate head injury. A guide to initial management. J Neurosurg 1992; 77: 562-4.

4. Colohan AR, Oyesiku NM. Moderate head injury: an overview. J Neurotrauma. 1992; 9 Suppl 1: S259-64.

5. Vitaz TW, et al. Outcome following moderate traumatic brain injury. Surgical Neurology. 2003;60: 285-91.

6. Einarsen CE, van der Naalt J, Jacobs B, Follestad T, Vik A, AK, Skandsen T. Moderate Traumatic Brain Injury: Clinical Characteristics and a Prognostic Model of 12-Month Outcome. World Neurosurg. 2018; 114: e1199-e1210.

7. González, M. Secuelas neuropsicológicas de los traumatismos craneoencefálicos. Anales de Psicología (2004). Vol 20: №2 (Diciembre).

8. Compagnone, C. Patients with moderate headinjury: A prospective multicenter study of 315 patients. Neurosurgery, 2009, April;64(4) 690-6.

9. Balwinder Singh et al. Meta-analysis of Glasgow Coma Scale and Simplified Motor Score in predicting traumatic brain injury outcomes, Brain Injury 2013; 27:3: 293-300.

10. Healey C, Osler TM, Rogers FB: Improving the Glasgow Coma Scale score: motor score alone is a better predictor.

11. Marshall LF, et al. A new classification of head injury based on computerized tomography. J Neurosurgery 1991;75 (Suppl):S14-20. JTrauma 54:671-680,2003.

12. Maas Al, et al. Prediction of outcome in traumatic brain injury with computed tomographic characteristics: a comparison between the computed tomographic classification and combinations of computed tomographic predictors. Neurosurgery. 2005;57:1173-82. 
13. Adams, JH, Doyle, D, et al. Diffuse axonal injury in head injury: definition, diagnosis and grading. Histopathology, 1989; jul, 15(1): 49-59.

14. Fabbri A. Early predictors of unfavourable putcome in subjects with moderate head injury in emergency department. J NeurolOio0'oeoru Neurosurgery and Psychiatry 2008; 79: 567573.

15. Kurland D, et al. Hemorrhagic progression of a contusion after traumatic brain injury: A Review. Journal Of Neurotrauma 29: 19 $-31.2012$.

16. Guylfoile, MR. Matrix Metalloproteinase Expression in Contusional Traumatic Brain Injury: A paired Microdialysis Study. Journal Of neurotrauma 32: 1553-1559. 2015.

17. Oertel $M$, et al. Progressive Hemorrhage after Head Trauma: Predictors and consequences of the evolving injury. J Neurosurg. 2002;96:109-16.

18. Poca MA, Sauquillo J, Baguena M, et al. Incidence of intracranial hypertension after severe head injury: a prospective study using the Traumatic Coma Data Bank classification. Acta Neurochir Suppl (Wien). 1998;71:27-30.

19. Ministerio de Salud. Guía Clínica Traumatismo Cráneo Encefálico moderado o grave. Santiago: Minsal, 2013.

20. Spaite D, et al. Mortality and Prehospital Blood Pressure in patients with major Traumatic Brain Injury. Implication for the Hypotension Threshold. JAMA Surgery; 2017, 152(4):360-368.

21. Keir, J. The impact of prehospital Ventilation on Outcome after Severe Traumatoc Brain Injury. Journal Of Trauma vol 62: 13301338. 2007.

22. Hawryluk GWJ, Aguilera S, Buki A, Bulger E, Citerio G, Cooper DJ, et al. A management algorithm for patients with intracranial pressure monitoring: the Seattle International Severe Traumatic Brain Injury Consensus Conference (SIBICC). Intensive Care Med. 2019; 45: 1783-1794.

23. Jacobs, B. Outcome prediction in moderated and Severe Traumatic Brain Injury: A focus on Computed Tomography Variables. Neurocritical Care /2013); 19:79-89.

24. Bullock, R. Surgical management of Traumatic Parenchimal
Lesions. Neurosurgery 2006;58: S2-46.

25. Mendelow, D. Early Surgery versus Initial Conservative Treatment in Patients with Traumatic Intracerebral Hemorrhage (STITCH). Journal of Neurotrauma 2015. 32: 1312-1323.

26. Oddo, M. Impact of tigh glycemic control on cerebral glucose metabolism after severe brain injury: a Microdialysis study. Critical Care Medicine. Dec; 36(12):3233-8. 2008.

27. Hessen, E. Influence of major AED on attention, reaction time, and speed of information processing. Epilepsia. Dec: 47(12): 2038-45. 2006.

28. Smith, KR. Neurobehavioral effects of phenytoin and carbamazepine in patients recovering from trauma. A comparative study. Arch Neurology Jul; 51: 653-60. 1994.

29. Lund, S. Moderate traumatic brain injury, acute phase course and deviations in physiological variables: an observational study. Scandinavian Journal of Trauma, Resuscitation and Emergency Medicine 2016; 24-77.

30. Fearnside $M$, McDougall $P$. Moderate head injury: a system of neurotrauma care. Aust NZJ Surg 1998; 68: 58-64.

31. Okonkwo, D. Brain Injury Optimization in Severe Traumatic Barin Injury Phase II: A phase II Ramdomized Trial. Critical Care Medicine, 2017; Volume 45 (11): 1907-1914.

32. Díaz-Arrastia, R. Molecular biomarkers in the neurological ICU: is there a role?. Curr Opin Crit Care 2020,26:000

33. Gordillo-Escobar, J.J. Usefulness of biomarkers in the prognosis of severe head injuries. Medicina Intensiva 2016; 40(2):105-112.

34. Agoston DV and Elsayed M. Serum-baed protein biomarkers in blast-induced traumatic brain injury spectrum disorder. Front. Neurol 2012; 3:107.

35. Thelin E. A serum protein biomarker panel improves outcomeprediction in human traumatic brain injury. J Neurotrauma2019; 36:2850-2862.

36. Godoy, D. Moderate Traumatic Barin Injury: The Grey Zone of Neurotrauma. Neurocritical Care 2016. Octubre (2):306-19.

37. Godoy, Aguilera Rabinstein. Potentially severe (Moderate) Traumatic Brain Injury: A New Categorization proposal. Critical Care Medicine.2020 Dec; 48(12) 1851-1854. 